\title{
Differential auxin transport and accumulation in the stem base lead to profuse adventitious root primordia formation in the aerial roots (aer) mutant of tomato (Solanum lycopersicum L.)
}

\author{
F. Mignolli ${ }^{\mathrm{a}, *}$, L. Mariotti ${ }^{\mathrm{b}}$, P. Picciarelli ${ }^{\mathrm{b}}$, M.L. Vidoz $^{\mathrm{a}, \mathrm{c}}$ \\ a Instituto de Botánica del Nordeste (IBONE), UNNE-CONICET, Sargento Cabral 2131, 3400 Corrientes, Argentina \\ b Dipartimento di Scienze Agrarie, Alimentari e Agro-ambientali, Università di Pisa, Via del Borghetto 80, 56124 Pisa, Italy \\ c Facultad de Ciencias Agrarias, UNNE, Sargento Cabral 2131, 3400 Corrientes, Argentina
}

\section{A R T I C L E I N F O}

\section{Article history:}

Received 17 October 2016

Received in revised form 13 February 2017

Accepted 23 February 2017

Available online 27 February 2017

\section{Keywords:}

Tomato

Aerial roots mutant

Adventitious roots

Polar auxin transport

IAA

\begin{abstract}
A B S T R A C T
The aerial roots (aer) mutant of tomato is characterized by a profuse and precocious formation of adventitious root primordia along the stem. We demonstrated that auxin is involved in the aer phenotype but ruled out higher auxin sensitivity of mutant plants. Interestingly, polar auxin transport was altered in aer, as young seedlings showed a reduced response to an auxin transport inhibitor and higher expression of auxin export carriers SIPIN1 and SIPIN3. An abrupt reduction in transcripts of auxin efflux and influx genes in older aer hypocotyls caused a marked deceleration of auxin transport in more mature tissues. Indeed, in 20 days old aer plants, the transport of labeled IAA was faster in apices than in hypocotyls, displaying an opposite trend in comparison to a wild type. In addition, auxin transport facilitators (SIPIN1, SIPIN4, SILAX5) were more expressed in aer apices than in hypocotyls, suggesting that auxin moves faster from the upper to the lower part of the stem. Consequently, a significantly higher level of free and conjugated IAA was found at the base of aer stems with respect to their apices. This auxin accumulation is likely the cause of the aer phenotype.
\end{abstract}

(c) 2017 Elsevier GmbH. All rights reserved.

\section{Introduction}

Adventitious roots (ARs) are post-embryonic roots originated from organs such as stems and leaves. They arise during normal plant development, as a response to environmental stress (e.g. flooding, wounding, nutrient deficiency) or following tissue culture regeneration of shoots (Li et al., 2009). ARs are ecologically important because they contribute to stabilize the soil in coastal or estuary regions and allow plant survival under biotic and abiotic stresses such as flooding (Steffens and Rasmussen, 2016). The economic importance of ARs in agriculture is prominent, since most horticultural and forestry practices rely on vegetative propagation to clone selected genotypes originated from breeding programs or already present in natural populations (De Klerk et al., 1999).

Although ARs can originate from different types of organs, they share some anatomical features since they always develop from cells adjacent to vascular bundles (Bellini et al., 2014). AR

\footnotetext{
* Corresponding author at: Laboratorio de Fisiología Vegetal, Instituto de Botánica del Nordeste- CONICET-UNNE, Sargento Cabral 2131, Corrientes, Argentina.

E-mail address: mignollif@virgilio.it (F. Mignolli).
}

development passes through at least three consecutive stages: induction, initiation and extension. Induction phase is characterized by molecular and biochemical events that precede any morphological changes, whereas during the initiation stage the organization of a root meristem occurs through coordinated cell divisions. Finally, the newly formed ARs extend across the cortical stem region and, when conditions are favorable for ARs to emerge, they elongate through epidermis in a process facilitated by programmed cell death of epidermal cells (Guan et al., 2015).

Auxin is virtually involved in every aspect of plant physiology and development (Delker et al., 2008). It influences and controls fruit formation (Dorcey et al., 2009), organ abscission (Basu et al., 2013), tropic response to light and gravity (Muday, 2001), and root development (Overvoorde et al., 2010). However, auxin effects not always follow a linear pathway where auxin acts alone, but it is often intricate because of tangling interactions with other phytohormones that participate in the same process (Sauer et al., 2013). Among auxin responses, the formation of ARs has been widely studied due to the technological implications that concern plant vegetative propagation (Blakesley, 1994). Indeed, auxins, as natural endogenous molecules or synthetic analogues, have a pivotal role in promoting AR formation during normal plant development 
or in cuttings of different species that require an induction to root (Pacurar et al., 2014b). Increased auxin content would promote AR formation. Rice seedlings that overexpressed of OsYUCCA1 gene, which seems to play an important role in IAA biosynthesis in this species, exhibited a supernumerary production of crown ARs (Yamamoto et al., 2007). Arabidopsis mutants with higher endogenous auxin are characterized by higher ability to develop ARs (Boerjan et al., 1995; Delarue et al., 1998). The superroot1 (sur1) mutant shows an abnormal proliferation of ARs along the hypocotyl that leads to the rupture of the cortex and epidermis (Boerjan et al., 1995). This mutant accumulates significantly high amounts of free and conjugated IAA as a result of a shift of the indole glucosinolates biosynthetic pathway towards IAA synthesis (Boerjan et al., 1995; Mikkelsen et al., 2004). Likewise, the excess of AR primordia in the superroot2 (sur2) mutant, is caused by an IAAoverproduction due to a dysfunctional cytochrome CYP83B1 that alters auxin homeostasis (Delarue et al., 1998; Barlier et al., 2000). On the other hand, mutations that negatively affect IAA biosynthesis are able to attenuate the "superroot" phenotype of sur2 when used as genetic background (Pacurar et al., 2014a).

Auxin is predominantly synthetized in young growing tissues such as stem apices, young leaves and developing seeds, and transported either undirectionally (phloematic) or directionally (Polar Auxin Transport, PAT) (Friml and Palme, 2002).The directional movement of auxin is facilitated by auxin transport carriers like AUXIN RESISTANT1/LIKE AUX1 (AUX1/LAX) auxin influx permeases, and PIN-FORMED (PIN) carrier proteins, responsible for the auxin efflux from cells (Blakeslee et al., 2005). In tomato, PIN and AUX/LAX genes are represented by 10 and 5 members, respectively. As in other dicots, they show similar phylogenetic organization and, in some cases, similar expression patterns (Pattison and Catalá, 2012). PAT is essential for the regulation of organ development, tissue elongation, and plastic response to the environment (Peer et al., 2011). PAT also plays a central role in the induction of AR development (Xu et al., 2005) since basipetal auxin transport along the stem contributes to the formation of AR primordia by favoring auxin accumulation in the rooting zone of cuttings (Da Costa et al., 2013). Indeed, blocking PAT either with specific inhibitors (Batten and Goodwin, 1981; Vidoz et al., 2010) or through genetic manipulation (Xu et al., 2005), results in an impaired AR initiation and emergence in different plant species.

Aerial roots (aer) tomato mutant is characterized by numerous AR primordia emerging from the stem base and was recently described for the first time in relation to its prompt ability to produce ARs in response to flooding stress (Vidoz et al., 2016). However, basic knowledge of the aer physiology underlying its profuse ARs primordia formation is lacking. Based on comparisons between aer and a non-mutated genotype, we propose that a reversed pattern of PAT regulation along aer stems accounts for auxin accumulation in epicotyls and hypocotyls, and this accumulation would induce the formation of abundant AR primordia.

\section{Materials and methods}

\subsection{Plant material and growth conditions}

Seeds of tomato (Solanum lycopersicum L.) cv. Ailsa Craig (AC, accession n. LA2838A) and the mutant aerial roots (aer, accession n. LA3205) were provided by the Tomato Genetics Resource Center (TGRC, University of California, Davis). The genetic background of aer is unknown and, therefore, we chose the AC cultivar as a control. AC has been used as a wild type in several physiological studies (Negi et al., 2010; Povero et al., 2011; Vidoz et al., 2010, 2016) and represents a contrasting genotype due to its lack of spontaneous AR primordia formation. Seeds were allowed to germinate

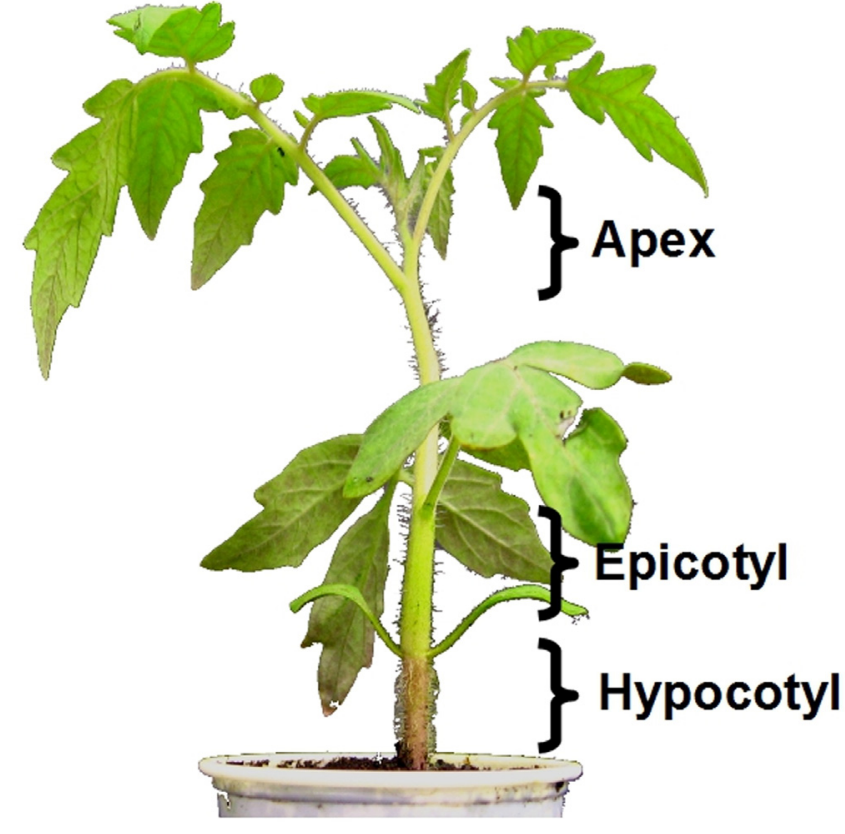

Fig. 1. Localization of tomato stem sections used for free and conjugated IAA detection and for auxin transport facilitator genes expression analysis.

on moist filter paper in petri dishes under light, with a temperature of $27 \pm 2{ }^{\circ} \mathrm{C}$. After five days from sowing, seedlings were transplanted in a peat-based substrate ( $\mathrm{pH}$ 5.5-6.5) amended with perlite (Dynamics 2 Q80, Buenos Aires, Argentina) in $220 \mathrm{ml}$ plastic pots. Plants were grown in a climatic chamber with a temperature of $26 \pm 1^{\circ} \mathrm{C}$ and a relative humidity that varied between 50 and $70 \%$. Light was supplied by high-pressure sodium lamps (Vialox ${ }^{\circledR}, 400 \mathrm{~W}$, OSRAM GmbH, Germany) with an intensity of $254 \mu \mathrm{mol} \mathrm{m}^{-2} \mathrm{~s}^{-1}$ at the plant height and a photoperiod of $15 \mathrm{~h}$. Plants were regularly irrigated with $1 / 4$ strength Hoagland solution. Plant samples, harvested for endogenous IAA determination and gene expression analysis, consisted of stem apices ( $1 \mathrm{~cm}$ long apical sections comprising the meristem), epicotyl sections $(0.3 \mathrm{~cm}$ long sections just above the cotyledons) and hypocotyl sections $(0.3 \mathrm{~cm}$ long sections above the root neck) (Fig. 1). Unless differently specified, samples were collected on 20-day-old plants.

\subsection{Microscopic observations}

AC and aer hypocotyls were collected from 10, 15, 20 and 25 days old seedlings. Hypocotyls were hand cut and cross sections were immersed in a safranine solution for $3 \mathrm{~min}$. Sections were rinsed 3 times with deionized water and observed with a stereomicroscope. Representative samples were photographed with a digital camera.

\subsection{Auxin and TIBA bioassays}

The auxin hypocotyl elongation assay was performed according to the method described by Kelly and Bradford (1986). Hypocotyl sections of $6.5 \mathrm{~mm}$ in length were excised from 5-day-old dark grown AC and aer seedlings and floated in a $2.5 \mathrm{mM} \mathrm{KH}_{2} \mathrm{PO}_{4}$ solution for $2 \mathrm{~h}$. Sections were placed in $6 \mathrm{~cm} \times 1.5 \mathrm{~cm}$ petri dishes and immersed in $10 \mathrm{ml}$ of basal medium $\left(2.5 \mathrm{mM} \mathrm{KH}_{2} \mathrm{PO}_{4} ; 2.5 \mathrm{mM} \mathrm{KCl}\right.$; $1 \mathrm{mM} \mathrm{Ca}\left(\mathrm{NO}_{3}\right)_{2} 4 \mathrm{H}_{2} \mathrm{O} ; 3 \%$ sucrose $)$. IAA was added to the basal medium in concentrations of $0,0.01,0.1,1,10$ and $100 \mu \mathrm{M}$. Sections were shaken constantly in an orbital shaker $(70 \mathrm{rpm})$ for $20 \mathrm{~h}$, at $27 \pm 2{ }^{\circ} \mathrm{C}$ under continuous light. Sections were then photographed and length was measured by processing the digital images with 
the public-domain software ImageJ (National Institutes of Health, http://rsb.info.nih.gov/ij).

For the auxin hypocotyl rooting assay, AC and aer seeds were surface sterilized (20\% v/v of commercial bleach, $0.1 \%$ Tween 20 for $10 \mathrm{~min}$ ) and sown on water-agar medium. After four days from germination, $1 \mathrm{~cm}$ long hypocotyl sections were excised and placed in MS medium enriched with increasing concentrations of the synthetic auxin 1-naphthaleneacetic acid (NAA, Sigma-Aldrich). Sections were cultivated for 3 weeks and the number of ARs was recorded.

The response of seedlings to auxin transport inhibition was tested by cultivating 3-day-old germinated seeds of AC and aer in MS medium enriched with increasing doses of TIBA $(2,3,5-$ triiodobenzoic acid, Sigma-Aldrich) for 7 days. Hypocotyl length was finally measured as described for the auxin hypocotyl elongation assay.

\subsection{TIBA and IAA stem application}

Fifty $\mu$ l of anhydrous lanolin supplemented with 1\% TIBA ( $1 \mu \mathrm{mol}$ per plant) were applied on 20 -day-old AC and aer plants. For controls, lanolin with the equivalent volume of ethanol used to dissolve the growth regulators was employed. The lanolin paste was applied as a band around the stem just above the cotyledonary node or the first true leaf insertion, depending on whether the effect on hypocotyl or epicotyl was considered, respectively. After 7 days from the treatment, AR primordia that were visible along the hypocotyl or epicotyl were counted.

\subsection{Extraction and analysis of free IAA}

Samples of stem apex, epicotyl and hypocotyl were homogenized in cold $70 \%(\mathrm{v} / \mathrm{v})$ aqueous acetone $(1: 5 \mathrm{w} / \mathrm{v})$ adding a known amount of $\left[{ }^{13} \mathrm{C}_{6}\right]$-IAA (Cambridge Isotopes Laboratories Inc., Andover, MA, USA) as internal standard. Homogenates were extracted 3 times and supernatants were collected after centrifugation at $4000 \mathrm{rpm}$ for $10 \mathrm{~min}$ at RT. The extraction solvents were reduced to the aqueous phase under vacuum, adjusted to $\mathrm{pH} 2.8$ and partitioned with diethyl ether. Diethyl ether extracts were dried under vacuum and resuspended in a small volume of $20 \%$ $(\mathrm{v} / \mathrm{v})$ aqueous methanol containing $0.01 \%$ (v/v) acetic acid. Sample extracts were purified by reverse-phase HPLC equipped with a Hypersil ODS column (Thermo) $150 \mathrm{~mm} \times 4.5 \mathrm{~mm}$ i.d. particle size $5 \mu \mathrm{m}$. Samples were eluted at a flow rate of $1 \mathrm{ml} \mathrm{min}^{-1}$ of $0.01 \%(\mathrm{v} / \mathrm{v})$ acetic acid in $20 \%(\mathrm{v} / \mathrm{v})$ methanol. The fraction corresponding to the elution volume of IAA standard was dried and silylated with N,Obis(trimethylsilyl) tri-fluoroacetamide (BSTFA) containing $1 \%$ of trimethylchlorosilane (Pierce, Rockford, IL, USA) at $70^{\circ} \mathrm{C}$ for $1 \mathrm{~h}$. IAA identification was carried out with Saturn 2200 quadrupole ion trap mass spectrometer coupled to a CP-3800 gas chromatograph (Varian Analytical Instruments, Walnut Creek, CA, USA) equipped with a MEGA 1 capillary column (MEGA, Legnano, Italy) $25 \mathrm{~m} \times 0.25 \mathrm{~mm}$ i.d. $\times 0.25 \mu \mathrm{m}$ film thickness, coated with $100 \%$ dimethylpolysiloxane. Quantification of IAA was accomplished by measuring the peak area ratio of dissociated ion $202(\mathrm{~m} / \mathrm{z})$ for IAA and ion 208 for the ${ }^{13} \mathrm{C}_{6}$-labeled internal standard.

\subsection{Extraction and analysis of conjugated IAA}

According to the method reported by Sorce et al. (2009), the residual aqueous phase after diethyl ether partition (see previous subsection) was pooled with the extracted pellet and hydrolyzed in $1 \mathrm{~N} \mathrm{NaOH}$ following the addition of a known amount of $\left[{ }^{13} \mathrm{C}_{6}\right]-$ IAA as internal standard. Hydrolysis was sustained for $1 \mathrm{~h}$ at $27^{\circ} \mathrm{C}$ in a capped vial continuously purged with helium. The extracts were centrifuged at $13,000 \mathrm{~g}$ for $30 \mathrm{~min}$ at $4{ }^{\circ} \mathrm{C}$ and the acidified supernatants were partitioned with diethyl ether as previously described. Again, the remaining aqueous phases were pooled with the pellet and hydrolyzed for $4 \mathrm{~h}$ at $100^{\circ} \mathrm{C}$ in $7 \mathrm{~N} \mathrm{NaOH}$ following the same procedure as before. Samples were processed to separate free IAA, subsequently purified and analyzed as detailed previously.

\subsection{IAA transport assay}

Basipetal IAA transport was assayed in apices and hypocotyls of 20-day-old AC and aer plants. Apices were cut in $2 \mathrm{~cm}$ long segments, removing meristems and leaflets. Subsequently, $2 \mu \mathrm{l}$ of $0.5 \mu \mathrm{g} \mu \mathrm{l}^{-1}\left[{ }^{13} \mathrm{C}_{6}\right]$-IAA dissolved in $2.5 \%$ ethanol were applied to the apical cut surface. In a similar way, $2 \mathrm{~cm}$ long segments were excised from the middle part of hypocotyls and labeled IAA was applied as described before. Segments were then incubated in a vertical position in a humid chamber for $4 \mathrm{~h}$. Following, apex and hypocotyl segments were cut in two halves (upper and lower) and immediately frozen.

Extraction and analysis of $\left[{ }^{13} \mathrm{C}_{6}\right]-\mathrm{IAA}$ in upper and lower portions of apex and hypocotyl segments were performed as previously specified. Peak area (abundance) of the $\left[{ }^{13} C_{6}\right]$-IAA dissociated ion $208(\mathrm{~m} / \mathrm{z})$ was measured in lower and upper halves and the percentage found in the lower half, that is, the basipetally transported fraction of $\left[{ }^{13} \mathrm{C}_{6}\right]-\mathrm{IAA}$, was calculated and diagrammed.

\subsection{Expression of auxin transport facilitator genes}

Total RNA was extracted as reported by Mignolli et al. (2015). Approximately $200 \mathrm{mg}$ of frozen stem apices, epicotyls and hypocotyls were ground to powder following the addition of $1 \mathrm{ml}$ of TRI Reagent ${ }^{\circledR}$ (MRC, Cincinnati, OH, USA). After centrifugation at $12000 \mathrm{~g}$ for $10 \mathrm{~min}$ at $4{ }^{\circ} \mathrm{C}$, the collected supernatant was partitioned with chloroform $(1: 0.2 \mathrm{v} / \mathrm{v})$. RNA was precipitated by adding ice-cold isopropanol $(1: 1 \mathrm{v} / \mathrm{v})$ and high salt solution ( $0.8 \mathrm{M}$ sodium citrate and $1.2 \mathrm{M}$ sodium chloride, Sigma-Aldrich) to the aqueous phase. RNA pellets were washed with $75 \%$ ethanol, dried, and solubilized in DEPC water. RNA purification from contaminating DNA was performed with the TURBO DNA free kit (Applied Biosystems/Ambion, Austin, TX, USA). Five $\mu g$ of total RNA were reverse transcribed into cDNA with the High-Capacity cDNA Archive Kit (Applied Biosystems).

Quantitative analysis of RNA transcripts of some auxin transport facilitator genes (SIPIN1, SIPIN3, SIPIN4, SILAX4 and SILAX5) was carried out using an ABI PRISM 7500 Real-Time PCR System (Applied Biosystems). Fifty ng of cDNA and SYBR ${ }^{\circledR}$ Green Master Mix (BioRad, Hercules, CA, USA) were used for amplification. Expression was normalized with transcript levels of the housekeeping gene LeEF1a.

\subsection{Gene accession numbers and primer pairs}

SIPIN1 (n. HQ127074) primer Fw 5'-TTATCATGGTGGAGCAGCAG-3', primer Rv 5'-TGTGCTTTGTTGCCATTGTT-3'. SIPIN3 (n. HQ127079) primer Fw 5'-AAGAAAATTGTGCTCAGGGC-3', primer Rv 5'-TGGTGCTGGTGCTGGTATTGT-3', SIPIN4 (n. HQ127078) primer Fw 5'-TAGGGGAATTTGGTGGTGAG-3', primer Rv 5'AGTCCAGTGGGTCCCTCTTT-3'. SILAX4 (n. HQ671066) primer Fw 5'-GCTGAGAAGCAAGCAGAGGA-3', primer Rv 5'-AAGCATCCCAAACAGAGCCA-3'. SILAX5 (n. HQ671067) primer Fw 5'-GCAGTAGAACAACCCCCAAGA-3', primer Rv 5'-CCAACCACCAAACCCAAAGC3'. LeEF1a (n. X53043) primer Fw 5'-CATCAGACAAACCCCTCCGT-3', Rv 5'-GGGGATTTTGTCAGGGTTGTAA-3'. 
A

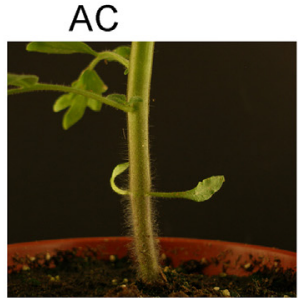

$10 d$
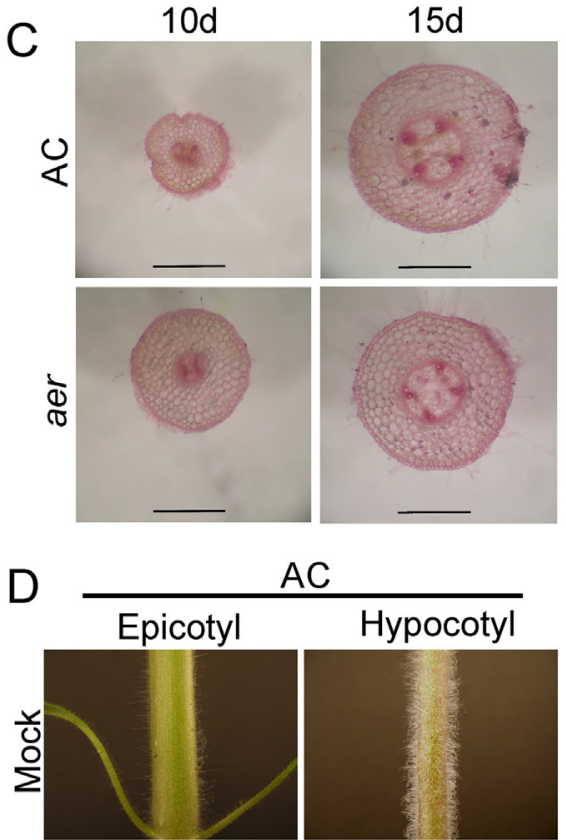

$\mathrm{AC}$

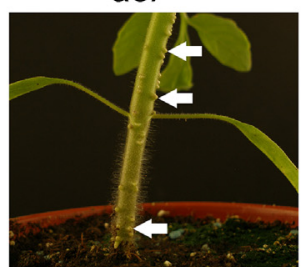

$15 d$
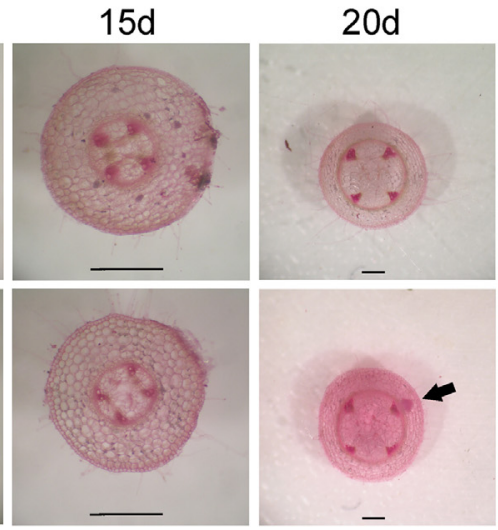

B

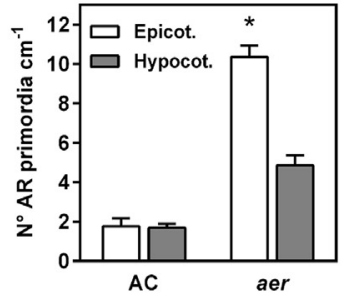

$25 d$
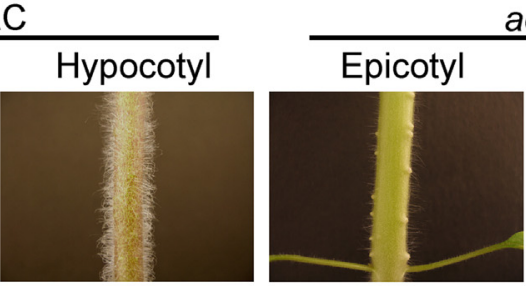

aer
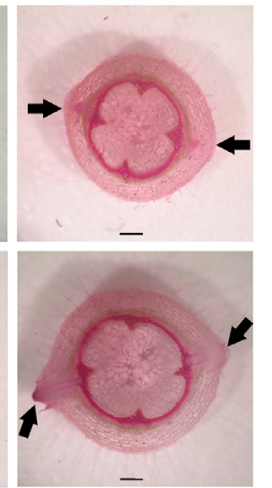
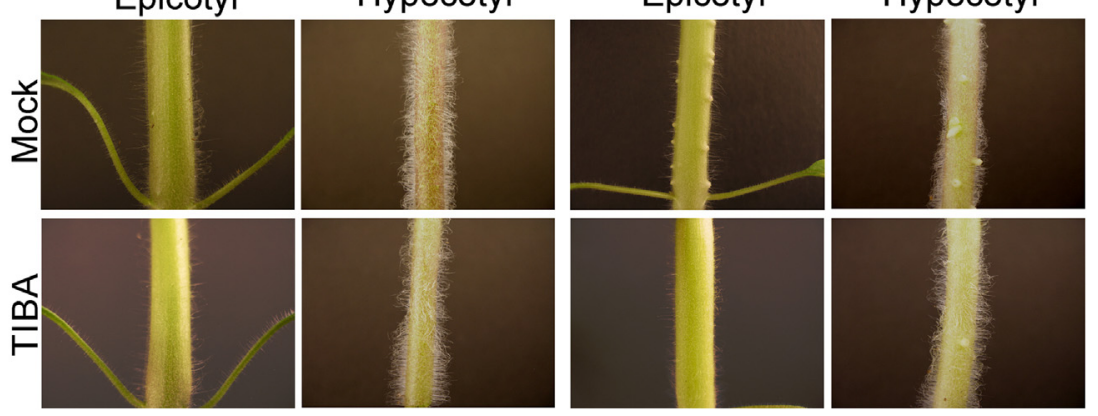

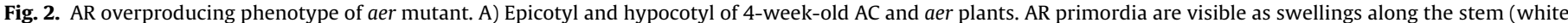

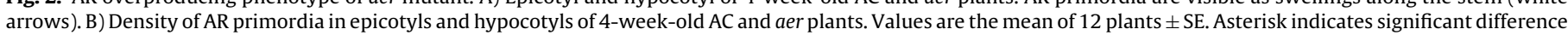

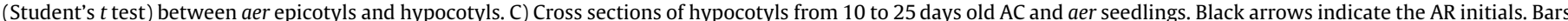

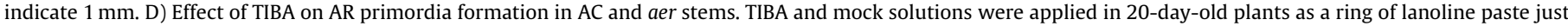
above the first true leaf node (for epicotyl treatment) or above the cotyledonary node (for hypocotyl treatment). Quantitative data for primordia are given in Table 1.

\subsection{Statistical analyses}

Comparison among more than two groups of samples were performed with one-way ANOVA analysis of variance with Tuckey's post test $(P<0.05)$. Student's $t$-test was adopted when comparing differences as in Figs. 2 B, 3 D and 4 D . All tests were carried out using the software Infostat version 2012 (Di Rienzo et al., 2012).

\section{Results}

\subsection{Precocious formation of AR primordia in aer hypocotyls}

The main phenotypic characteristic of aer plants is the presence of numerous AR primordia that emerge from the surface of epicotyls and hypocotyls and are clearly visible in 4-week-old seedlings (Fig. 2A). Although primordia protrude, they arrest their growth before causing epidermis rupture. The density of AR primordia in epicotyls and hypocotyls of aer was almost 6 and 3 folds higher than in AC, respectively (Fig. 2B). To note, while the density of AR primordia was similar in epicotyls and hypocotyls of AC plants, in aer epicotyls it almost doubled the density observed in hypocotyls (Fig. 2B).

In order to ascertain the plant age at which AR primordia formation starts in AC and aer plants, cross sections of hypocotyls from 10-, 15-, 20- and 25-day-old seedlings were observed (Fig. 2C). Microphotographs of representative samples showed that in aer, primordia were more precociously formed with respect to AC, starting to emerge from the pericycle when plants were 20 days old. In 25 days old plants, primordia were visible also in AC stems but those found in aer had elongated more through the cortex (Fig. 2C).

\subsection{TIBA blocked the formation of AR primordia at the base of aer stems}

Following, we aimed to determine the role of auxin in AR primordia formation of the aer mutant. Auxin transport towards the basal part of the stem was inhibited by applying the auxin efflux inhibitor TIBA. One percent TIBA almost completely abolished the emergence of AR primordia in AC and aer epicotyls and hypocotyls (Fig. 2D; Table 1) but in the mutant hypocotyls this decrease appeared less severe than in epicotyls ( 7.5 vs 191 fold decrease). 

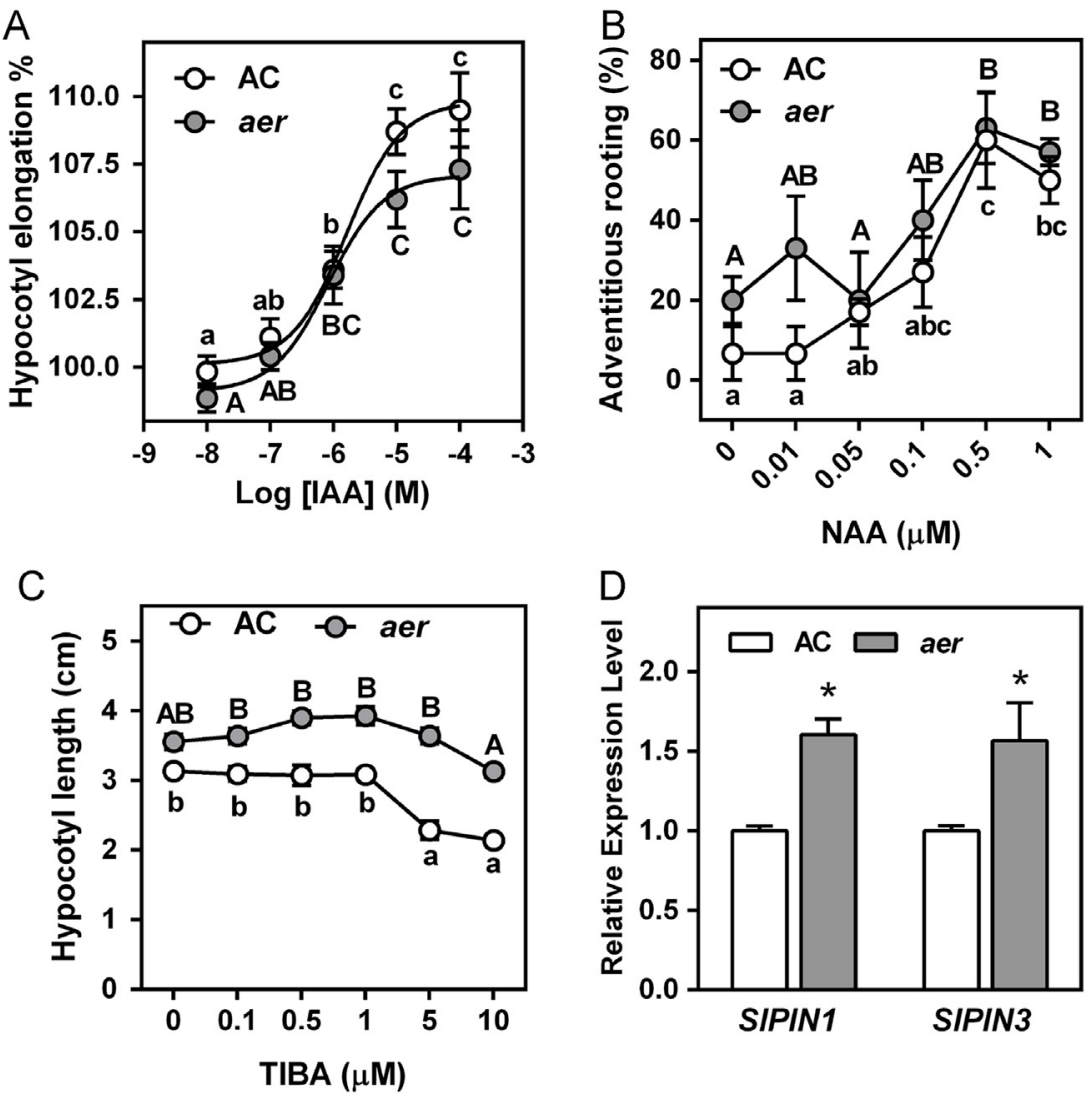

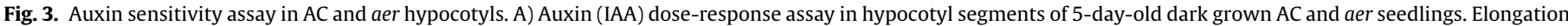

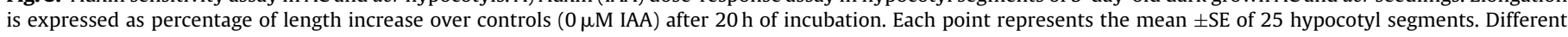

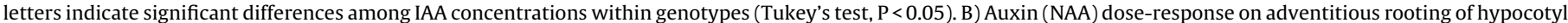

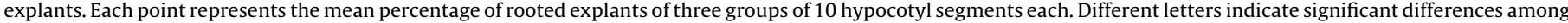

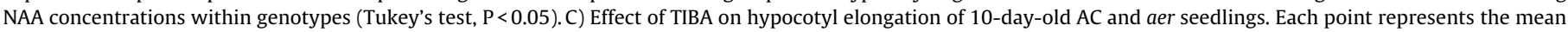

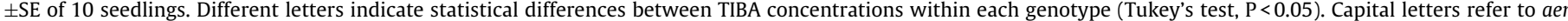

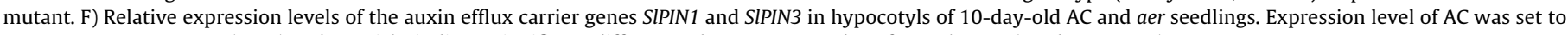
one. Data are mean $\pm S D(n=3)$ and asterisks indicate significant differences between AC and aer for each gene (Student's $t$ test).

Table 1

Density of AR primordia ( $\mathrm{n}^{\circ}$ of primordia $\mathrm{cm}^{-1}$ ) in epicotyls and hypocotyls of AC and aer plants treated with mock and $1 \%$ TIBA. Values are means \pm SE, numbers of replicates between brackets.

\begin{tabular}{llllll}
\hline & AC & & \multicolumn{2}{l}{ aer } \\
\cline { 2 - 3 } \cline { 5 - 6 } \cline { 5 - 6 } & Epicotyl & Hypocotyl & & Epicotyl & Hypocotyl \\
\hline Mock & $0.70 \pm 0.26(10)$ & $0.85 \pm 0.11(10)$ & & $9.54 \pm 0.64(13)$ & $3.13 \pm 0.61(12)$ \\
TIBA & n.d. (9) & n.d.(10) & & $0.05 \pm 0.03(13)$ & $0.42 \pm 0.15(12)$ \\
\hline
\end{tabular}

\subsection{Aer mutation does not increase auxin responsiveness but alters auxin transport}

In order to establish whether the formation of AR primordia in aer is exerted by hormone concentration changes, by alterations in tissue sensitivity or by a combination of both, we examined auxin dose response on elongation of hypocotyl segments and their rooting ability. AC and aer hypocotyls elongated in presence of IAA yielding a sigmoidal curve (Fig. 3A). Although both AC and aer reached the maximum elongation at $10^{-5} \mathrm{M}$ IAA and no further increase was measured at $10^{-4} \mathrm{M}$ IAA, the increase in elongation observed between $10^{-6}$ and $10^{-5} \mathrm{M}$ was smaller in aer than in AC.

With respect to auxin-induced rooting ability, segments excised from 7-day-old AC and aer hypocotyls were able to regenerate ARs in presence of NAA in a dose dependent manner (Fig. 3B). The percentage of rooting in both genotypes was significantly higher than in controls only when NAA concentration in the medium was $0.5 \mu \mathrm{M}$, producing about $60 \%$ of rooted hypocotyls.

In order to test whether PAT was altered in the aer mutant with respect to AC, seedlings were grown in presence of increasing concentrations of TIBA. Interestingly, hypocotyl growth in AC was inhibited when TIBA concentration was $5 \mu \mathrm{M}$, with a reduction in length of $27 \%$ respect to the control (Fig. 3 C). In aer, only hypocotyls exposed to $10 \mu \mathrm{M}$ TIBA were significantly shorter than those exposed to lower concentrations (Fig. 3C). In addition, the auxin efflux carrier genes SIPIN1 and SIPIN3 in 10 days old seedlings were more expressed in aer hypocotyls than in AC, further supporting the hypothesis of an altered auxin transport in $\operatorname{aer}$ (Fig. 3D).

\subsection{IAA accumulates in basal region of aer stems}

Analysis of endogenous free and conjugated IAA was performed considering three portions of AC and aer stems: apex, epicotyl and hypocotyl. Data revealed that in AC, free IAA accumulated in the apex concomitantly with a high amount of conjugated IAA forms (IAA-ester and IAA-amide) (Fig. 4A). Older tissues showed a progressive reduction in free IAA with the lowest value found in the hypocotyl. Conversely, in the aer mutant the highest amount of free IAA was found in the epicotyl whereas the level measured in the hypocotyl was similar to the apex (Fig. 4B). Notably, the concentration of free IAA in aer hypocotyls was more than two times higher than in AC. The distribution pattern of conjugated IAA was similar in both genotypes. Although the highest content of IAA-amide was 
A

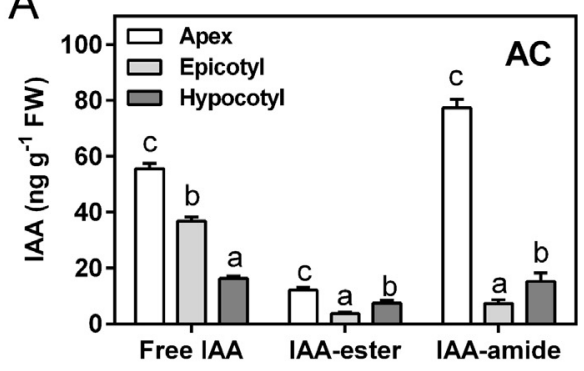

B

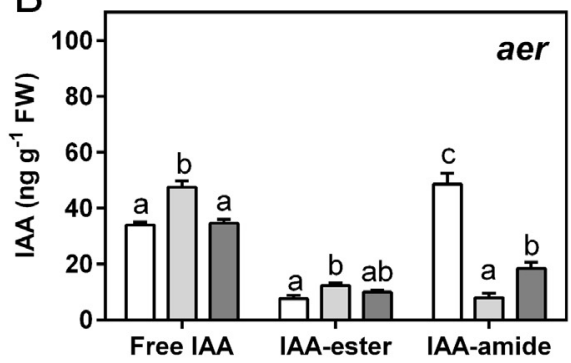

C
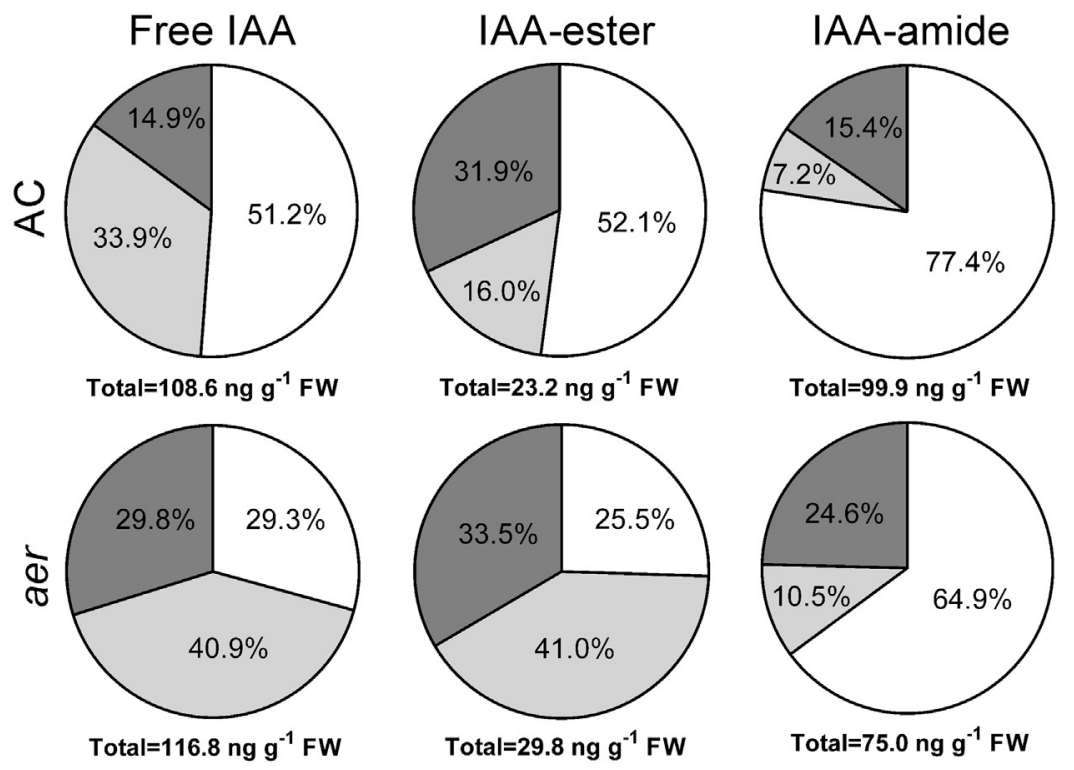

A
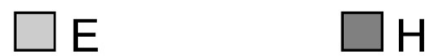

D

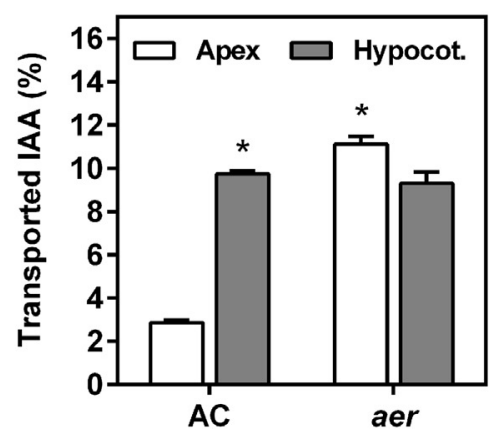

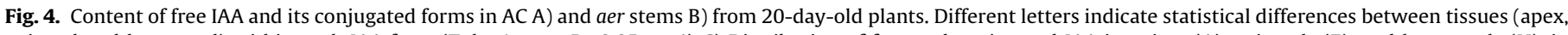

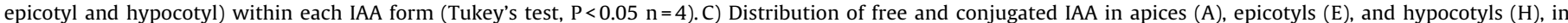

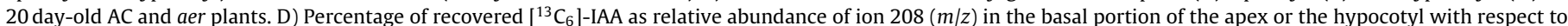

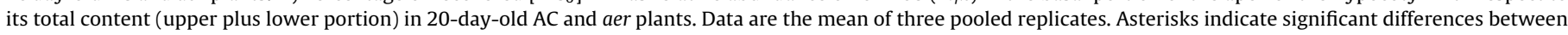
apices and hypocotyls for each genotype (Student's $t$ test).

found in the apex, it was 38\% lower in the mutant than in AC (Fig. 4A, $B)$. The distribution of free and conjugated IAA content in AC and aer plants clearly showed that while in AC all IAA forms are prevalently concentrated in the apex (51.2, 52.1 and $77.4 \%$ for free, ester- and amide IAA respectively), in aer, epicotyls and hypocotyls accumulated more than 70 and $74 \%$ of free IAA and IAA-ester, respectively (Fig. 4C).

\subsection{IAA is more slowly transported in aer hypocotyls than in apices}

Following, we aimed to determine whether the accumulation of free IAA at the base of aer plants is due to a distinct IAA transport along stems. The basipetal transport was quantified by measuring the proportion of $\left[{ }^{13} \mathrm{C}_{6}\right]$-IAA that was polarly transported from the point of application towards the lower portion of tissue. The 
percentage of transported $\left[{ }^{13} \mathrm{C}_{6}\right]$-IAA was significantly lower in the apex (2.9\%) than in the hypocotyl (9.7\%) of AC, whereas an opposite proportion between the two tissues was observed in aer (11.1 vs 9.3\% for apex and hypocotyl respectively, $\mathrm{P}<0.05$ ) (Fig. 4D). These data indicate that IAA is transported more slowly in AC apices than in hypocotyls, while it moves more rapidly in apices than in hypocotyls of the aer mutant.

\subsection{PIN and AUX/LAX genes expression sharply decrease in aer during hypocotyl development}

In order to observe whether the AR primordia appearance in aer is accompanied by a change in the expression of PIN and AUX/LAX genes during hypocotyl development, transcript levels of SIPIN1, SIPIN4, SILAX4 and SILAX5 were monitored in hypocotyls collected from 10, 15, 20 and 25 days old plants (Fig. 5). In both AC and aer, hypocotyls of 10-day-old plants showed the highest expression level of all analyzed genes. In both genotypes, gene expression dramatically decreased in 15-day-old hypocotyls and was maintained at low levels also in hypocotyls from 20-day-old plants. However, it is worth noting that this decrease in PIN and AUX/LAX transcript abundance was steeper in the mutant between 10- and 20-day-old hypocotyls (when AR primordia start to appear). Indeed, relative expression levels declined 500 vs 31 folds for SIPIN1, 125 vs 18 for SIPIN4, 8.3 vs 2.5 for SILAX4 and 67 vs 5.0 for SILAX5 in aer and AC, respectively. In 25-day-old AC hypocotyls, an increase in expression levels of SIPIN1, SILAX4 and SILAX5 was observed (Fig. 5).

\subsection{PIN and AUX/LAX genes are less induced in the basal part of aer stems than in apices}

To further explore IAA transport in AC and aer stems, the transcript analysis of several auxin transport facilitator genes was performed on apices, epicotyls and hypocotyls of 20-day-old plants (Fig. 6). In AC, SIPIN1, SIPIN4 and SILAX4 genes were more expressed in hypocotyls, being over 10, 2.5 and 12 times more abundant than in the apex. With an opposite pattern, in aer, the highest transcript levels for SIPIN1, SIPIN4 and SILAX5 genes was observed in the apex whereas the lowest levels were found in the hypocotyl (12-, 3.5and 4.1-fold lower, respectively) (Fig. 6).

\section{Discussion}

To date, aer is the only tomato mutant that has been reported to show an AR overproducing phenotype (Fig. 2A, B) (Vidoz et al., 2016). In aer, AR primordia start to be visible 5 days earlier than in AC (Fig. 2C) and are originated from the pericycle (Byrne et al., 1975). By virtue of this precocity, the mutant proved to be more tolerant to flooding stress since it is able to rapidly reconstitute a functional AR system (Vidoz et al., 2016). In order to take a first step towards the physiological characterization of the mutant, we assessed whether the abundant AR primordia formation is dependent on auxin, as its involvement in AR formation is widely known (Pacurar et al., 2014b). The inhibition of basipetal auxin transport through TIBA application dramatically reduced the appearance of primordia in aer epicotyls (Fig. 2D; Table 1). In hypocotyls, this decrease was less pronounced than in epicotyls possibly due to the presence of already formed primordia at the moment of the treatment of hypocotyl tissues (Fig. 2C, D; Table 1). These data indicated that in aer, AR primordia formation is strictly dependent on the polarly transported auxin from younger to older parts of the stem.

Since increased auxin sensitivity correlates with tissue rhizogenic ability (Visser et al., 1996; Spanò et al., 1988), we evaluated whether the aer phenotype is caused by increased auxin responsiveness in the stem. However, data revealed that $1 \mu \mathrm{M}$ IAA was able to induce a similar hypocotyl elongation in both $A C$ and aer (Fig. 3A) and $0.5 \mu \mathrm{M}$ NAA equally promoted AR formation in hypocotyl explants, (Fig. 3B) indicating that hypocotyls of both genotypes have comparable auxin sensitivity.

Auxin transport has been frequently associated with rooting ability of cuttings (Garrido et al., 2002; López Nicolás et al., 2004). In Petunia, auxin accumulation at the rooting zone via PAT is probably responsible for the establishment of a new sink, which in turn induces cell divisions that lead to the formation of new AR meristems (Ahkami et al., 2013). Taking this into account, it was hypothesized that the aer mutation may alter PAT. When aer seedlings were grown in presence of increasing concentrations of TIBA, they showed a lower inhibition of hypocotyl elongation (Fig. 3C). The higher level of SIPIN1 and SIPIN3 expression in aer seedlings (Fig. 3D), may confer partial resistance to the inhibitory effect of TIBA, since lower sensitivity to PAT inhibitors was inversely correlated with PINs transcript levels (Xu et al., 2005; Chen et al., 2012).

Auxin is predominantly synthesized in shoot apices and is directed basipetally by the action of auxin influx and efflux transporters (Muday and DeLong, 2001). Since the modulation of auxin carrier genes expression reflects changes in auxin transport (Peer et al., 2004), we examined the relative transcript abundance of SIPIN1, SIPIN4, SILAX4 and SILAX5, whose expression has been previously reported in tomato vegetative parts (Nishio et al., 2010; Kharshiing et al., 2010; Pattison and Catalá, 2012).

The expression levels of SIPIN1, SIPIN4, SILAX4 and SILAX5 (Fig. 5), suggest that the auxin transport capacity of hypocotyls declines with age in accordance with a previous report by Suttle (1991). However, in aer, this decrease seems to be more drastic especially between 10 and 20 days old seedlings coinciding with the early initiation of AR primordia (Fig. 2C). It has been proposed that the PAT slowdown determines the accumulation of auxin at the shoot base and is therefore primarily responsible for AR formation in response to flooding (Visser et al., 1996). The accumulation of auxin transport inhibitors, such as flavonoids, in older tissues has been proposed as one of the causes of auxin transport reduction with increasing plant age (Sánchez-Bravo et al., 1992). However, this does not seem the case for aer considering that a lower quantity of total flavonoids was detected in epicotyls and hypocotyls (data not shown).

We also investigated whether there is an alteration in basipetal auxin transport along the stem of aer plants at the moment of primordia formation (20 days old seedlings). When auxin basipetal transport was measured in apices and hypocotyls, an opposite trend between AC and aer was observed (Fig. 4D). In AC, the amount of labeled IAA recovered in the basal part of the apex was relatively lower than in the basal part of the hypocotyl, suggesting a slower IAA movement in the apex. This is in accordance with the data reported by Kojima et al. (2002) where IAA diffused more slowly in the apex than in a more basal portion of tomato stem. Interestingly, in aer, a smaller content of labeled IAA was found in the basal part of the hypocotyl in comparison to the basal part of the apex, indicating that IAA was transported at a slower rate in the hypocotyl with respect to the apex (Fig. 4D).

Consistent with the IAA transport data, the expression pattern of SIPIN1, SIPIN4, SILAX4 and SILAX5 in the apex and at the base of aer stems with respect to AC (Fig. 6) support the idea that, in the mutant, the quantity of IAA transported from the apex to the base would be higher and the capacity of draining IAA towards roots would be lower than in AC. The slower auxin transport in AC apex with respect to the more basal stem portion results in the accumulation of higher amounts of free IAA and the consequent formation of more conjugated IAA in this tissue (Zazimalova and Napier, 2003) (Fig. 4A-C). On the contrary, in aer plants, free and IAA-ester are predominantly concentrated in the epicotyl and hypocotyl, where auxin transport is slower. To note, the amount of 
$\mathrm{AC}$
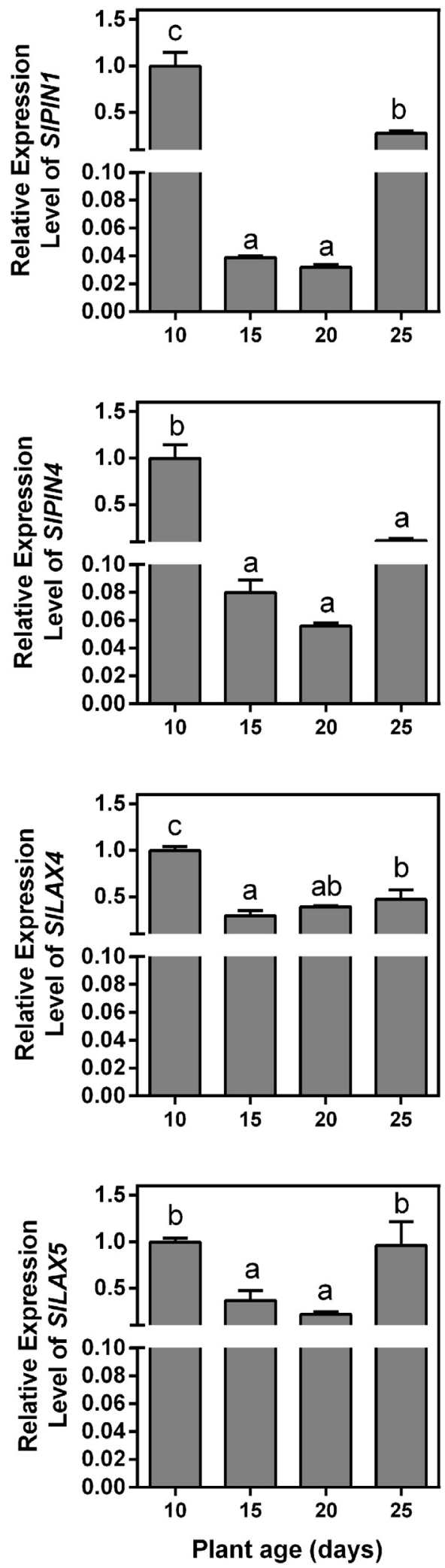

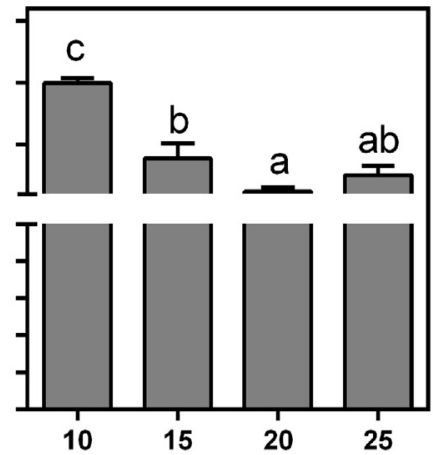

aer
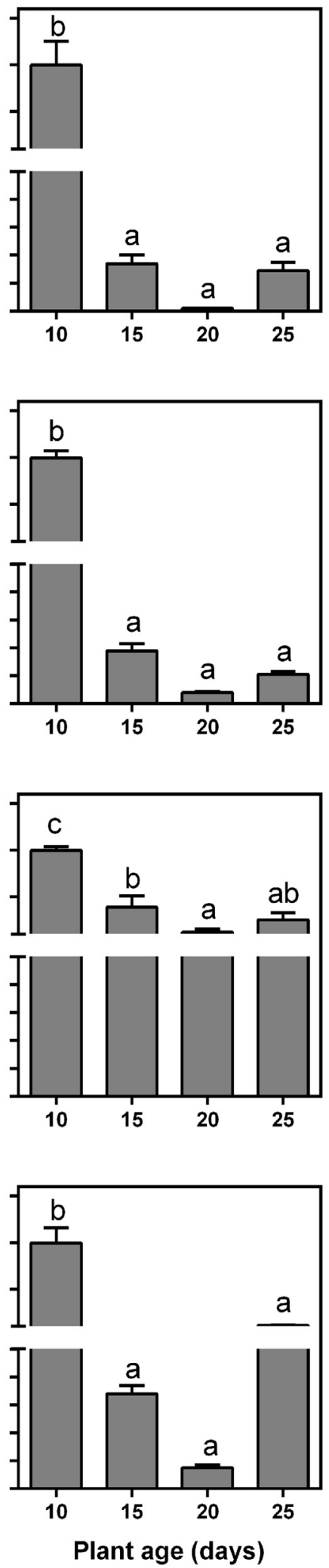

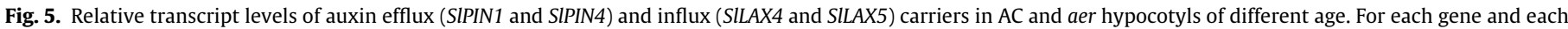

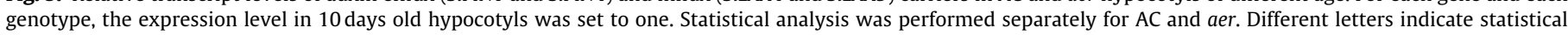
differences according to Tuckey's test $(P<0.05 n=3)$. 
$\mathrm{AC}$
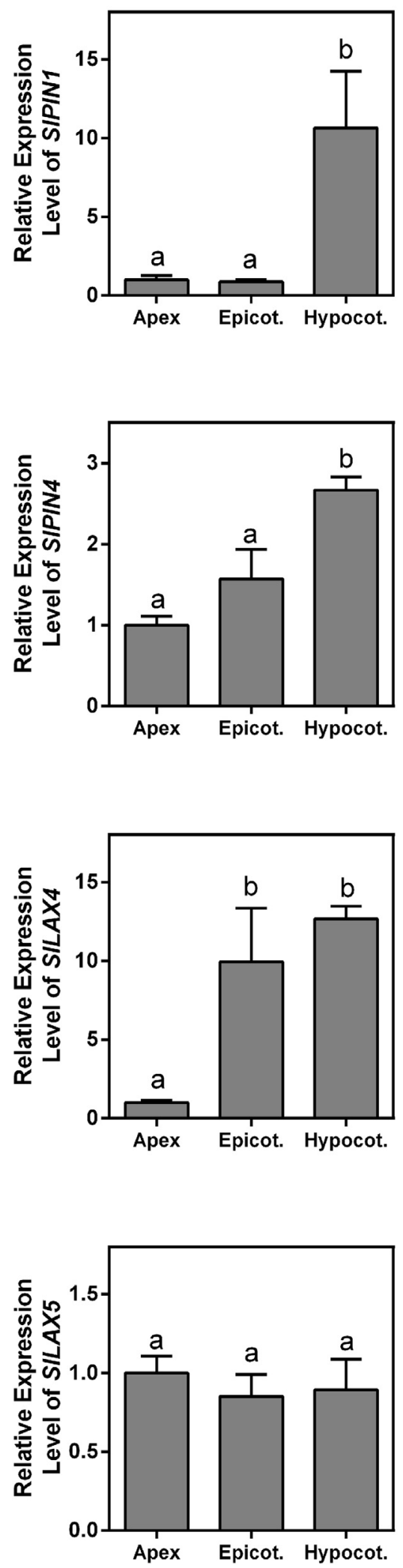

aer
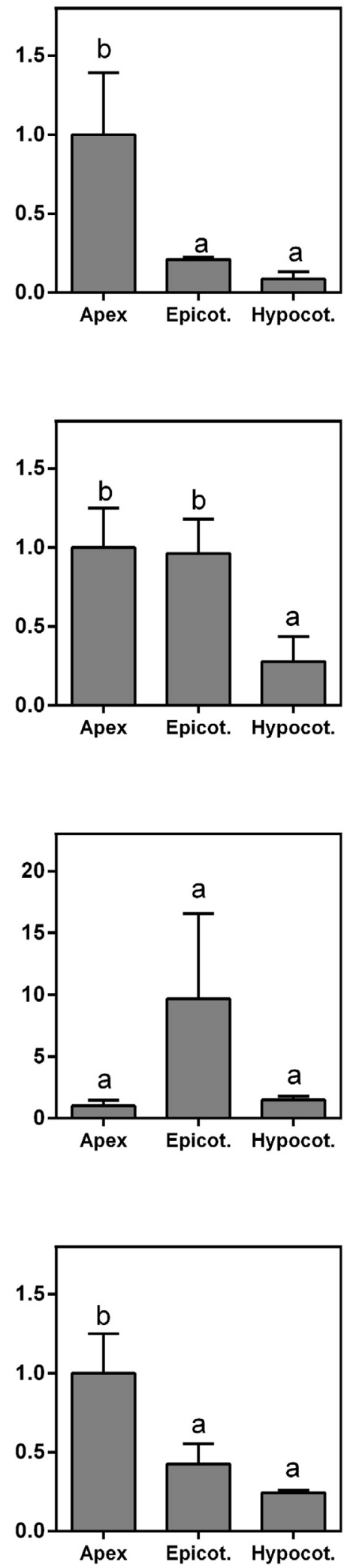

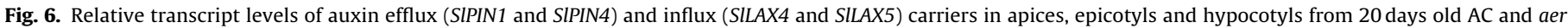

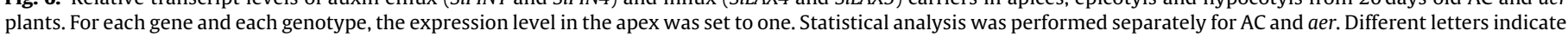
statistical differences according to Tuckey's test $(P<0.05 n=3)$. 


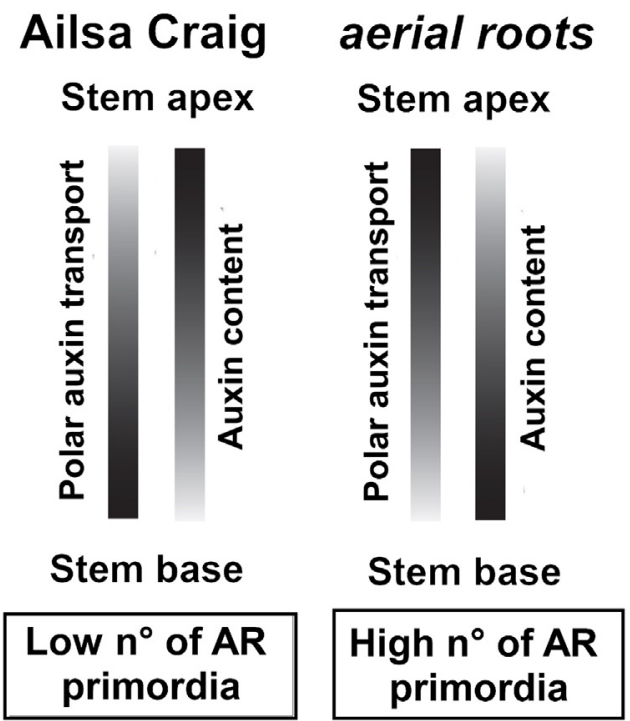

Fig. 7. Schematic representation of the proposed hypothesis for aer mutant phenotype. Abundant formation of AR primordia in the mutant stem base is probably caused by a reduction of basipetally transported auxin from the apex towards more basal stem tissues (epicotyl and hypocotyl). Decreased PAT would determine auxin accumulation, which in turn induces AR primordia formation.

total free IAA is similar in both genotypes (Fig. 4C) supporting the idea that differential IAA distribution is due to the altered auxin transport capacity of the aer stem rather than a higher free IAA synthesis in the mutant. The presence of a decreased basipetal auxin transport from the apex to the hypocotyl in aer may produce what has been described as "barrier effect" (Sánchez-Bravo et al., 1992). This phenomenon occurs when the amount of auxin that moves from the upper zone of a tissue exceeds the transport capacity of a more basal portion, resulting in its accumulation in those tissues with lower auxin transport capacity.

Although the genetic nature of the aer mutation has not been deciphered yet, it is supposed that more than one gene may be required for the expression of its phenotype, or that epistasis may be involved (Roger Chetelat, personal communication). In any case, our data sustain the hypothesis that the AR overproducing phenotype of aer seems strictly linked to a different distribution of IAA along the stem. In fact, the higher auxin transport in apices than in basal stem portions is suggested to be the cause of IAA accumulation in aer epicotyls and hypocotyls, which in turn would be the stimulus for the abundant AR primordia formation (Fig. 7). We believe that our work has shed light over the auxin-driven mechanism that causes the early formation of AR primordia, making aer a useful system for studies regarding auxin transport regulation, especially if the nature of the mutation is identified.

\section{Acknowledgement}

Our work was funded by SGCyT-UNNEPI-2011-P001 (General Secretariat of Science and Technology, UNNE).

\section{References}

Ahkami, A.H., Melzer, M., Ghaffari, M.R., Pollmann, S., Ghorbani Javid, M., Shahinnia, F., Hajirezaei, M.R., Druege, U., 2013. Distribution of indole-3-acetic acid in Petunia hybrida shoot tip cuttings and relationship between auxin transport, carbohydrate metabolism and adventitious root formation. Planta 238, 499-517.

Barlier, I., Kowalczyk, M., Marchant, A., Ljung, K., Bhalerao, R., Bennett, M. Sandberg, G., Bellini, C., 2000. The SUR2 gene of Arabidopsis thaliana encodes the cytochrome P450 CYP83B1, a modulator of auxin homeostasis. Proc. Natl. Acad. Sci. U. S. A. 97, 14819-14824.
Basu, M.M., González-Carranza, Z.H., Azam-Ali, S., Tang, S., Shahid, A.A., Roberts, J.A., 2013. The manipulation of auxin in the abscission zone cells of Arabidopsis flowers reveals that indoleacetic acid signaling is a prerequisite for organ shedding. Plant Physiol. 162, 96-106.

Batten, D.J., Goodwin, P.B., 1981. Auxin transport inhibitors and the rooting of hypocotyl cuttings from etiolated mung-bean Vigna radiata (L.) Wilczek seedlings. Ann. Bot. Lond. 47, 497-503.

Bellini, C., Pacurar, D.I., Perrone, I., 2014. Adventitious roots and lateral roots: similarities and differences. Annu. Rev. Plant Biol. 65, 639-666.

Blakeslee, J.J., Peer, W.A., Murphy, A.S., 2005. Auxin transport. Curr. Opin. Plant Biol. 8, 494-500.

Blakesley, D., 1994. Auxin metabolism and adventitious root formation. In: Davis, T.D., Haissig, B.E. (Eds.), Biology of Adventitious Root Formation. Plenum Press, New York, pp. 143-151.

Boerjan, W., Cervera, M.T., Delarue, M., Beeckman, T., Dewitte, W., Bellini, C. Caboche, M., van Onckelen, H., van Montagu, M., Inzé, D., 1995. Superroot, a recessive mutation in Arabidopsis, confers auxin overproduction. Plant Cell 7, 1405-1419.

Byrne, J.M., Collins, K.A., Cashau, P.F., Aung, L.H., 1975. Adventitious root development from seedling hypocotyl of Lycopersicon esculentum. Am. J. Bot. 62, 731-737.

Chen, Y., Fan, X., Song, W., Zhang, Y., Xu, G., 2012. Over-expression of OsPIN2 leads to increased tiller numbers, angle and shorter plant height through suppression of OsLAZY1. Plant Biotechnol. J. 10, 139-149.

Da Costa, C.T., de Almeida, M.R., Ruedell, C.M., Schwambach, J., Maraschin, F.S., Fett-Neto, A.G., 2013. When stress and development go hand in hand: main hormonal controls of adventitious rooting in cuttings. Front. Plant Sci. 4, 133.

De Klerk, G.J., van der Krieken, W., de Jong, J.C., 1999. Review the formation of adventitious roots: new concepts, new possibilities. In Vitro Cell Dev. Plant 35 189-199.

Delarue, M., Prinsen, E., van Onckelen, H., Caboche, M., Bellini, C., 1998. Sur2 mutations of Arabidopsis thaliana define a new locus involved in the control of auxin homeostasis. Plant J. 14, 603-611.

Delker, C., Raschke, A., Quint, M., 2008. Auxin dynamics: the dazzling complexity of a small molecule's message. Planta 227, 929-941.

Di Rienzo, J.A., Casanoves, F., Balzarini, M.G., Gonzalez, L., Tablada, M., Robledo, C.W., InfoStat versión, 2012. InfoStat Group, Facultad De Ciencias Agropecuarias. Universidad Nacional de Córdoba, Aergentina http://www. infostat.com.ar.

Dorcey, E., Urbez, C., Blázquez, M.A., Carbonell, J., Perez-Amador, M.A., 2009 Fertilization-dependent auxin response in ovules triggers fruit development through the modulation of gibberellin metabolism in Arabidopsis. Plant J. 58, 318-332.

Friml, J., Palme, K., 2002. Polar auxin transport-old questions and new concepts? Plant Mol. Biol. 49, 273-284.

Garrido, G., Ramón Guerrero, J., Angel Cano, E., Acosta, M., Sánchez-Bravo, J., 2002. Origin and basipetal transport of the IAA responsible for rooting of carnation cuttings. Physiol. Plant. 114, 303-312.

Guan, L., Murphy, A.S., Peer, W.A., Gan, L., Li, Y., Cheng, Z.M., 2015. Physiological and molecular regulation of adventitious root formation. CRC. Crit. Rev. Plant Sci. 34, 506-521 (Max).

Kelly, M.O., Bradford, K.J., 1986. Insensitivity of the diageotropica tomato mutant to auxin. Plant Physiol. 82, 713-717.

Kharshiing, E.V., Kumar, G.P., Ditengou, F.A., Li, X., Palme, K., Sharma, R., 2010. The polycotyledon (pct1-2) mutant of tomato shows enhanced accumulation of PIN1 auxin transport facilitator protein. Plant Biol. 12, 224-228.

Kojima, K., Ohtake, E., Yu, Z., 2002. Distribution and transport of IAA in tomato plants. Plant Growth Regul. 37, 249-254.

López Nicolás, J.I., Acosta, M., Sánchez-Bravo, J., 2004. Role of basipetal auxin transport and lateral auxin movement in rooting and growth of etiolated lupin hypocotyls. Physiol. Plant. 121, 294-304.

Li, S.W., Xue, L., Xu, S., Feng, H., An, L., 2009. Mediators, genes and signaling in adventitious rooting. Bot. Rev, 75, 230-247.

Mignolli, F., Vidoz, M.L., Mariotti, L., Lombardi, L., Picciarelli, P., 2015. Induction of gibberellin 20-oxidases and repression of gibberellin 2-oxidases in unfertilized ovaries of entire tomato mutant, leads to accumulation of active gibberellins and parthenocarpic fruit formation. Plant Growth Regul. 75, 415-425.

Mikkelsen, M.D., Naur, P., Halkier, B.A., 2004. Arabidopsis mutants in the C-S lyase of glucosinolate biosynthesis establish a critical role for indole-3-acetaldoxime in auxin homeostasis. Plant J. 37, 770-777.

Muday, G.K., DeLong, A., 2001. Polar auxin transport: controlling where and how much. Trends Plant Sci. 6, 535-542.

Muday, G.K., 2001. Auxins and tropism. J. Plant Growth Regul. 20, 226-243.

Negi, S., Sukumar, P., Liu, X., Cohen, J.D., Muday, G.K., 2010. Genetic dissection of the role of ethylene in regulating auxin-dependent lateral and adventitious root formation in tomato. Plant J. 61, 3-15.

Nishio, S., Moriguchi, R., Ikeda, H., Takahashi, H., Takahashi, H., Fujii, N., Guilfoyle, T.J., Kanahama, K., Kanayama, Y., 2010. Expression analysis of the auxin efflux carrier family in tomato fruit development. Planta 232, 755-764.

Overvoorde, P., Fukaki, H., Beeckman, T., 2010. Auxin control of root development Cold Spring Harbor Perspect. Biol. 2, a001537.

Pacurar, D.I., Pacurar, M.L., Bussell, J.D., Schwambach, J., Pop, T.I., Kowalczyk, M., Gutierrez, L., Cavel, E., Chaabouni, S., Ljung, K., Fett-Neto, A.G., Pamfil, D., Bellini, C., 2014a. Identification of new adventitious rooting mutants amongst suppressors of the Arabidopsis thaliana superroot2 mutation. J. Exp. Bot. 65, 1605-1618. 
Pacurar, D.I., Perrone, I., Bellini, C., 2014b. Auxin is a central player in the hormone cross-talks that control adventitious rooting. Physiol. Plant. 151, 83-96.

Pattison, R.J., Catalá, C., 2012. Evaluating auxin distribution in tomato (Solanum lycopersicum) through an analysis of the PIN and AUX/LAX gene families. Plant J. 70, 585-598

Peer, W.A., Bandyopadhyay, A., Blakeslee, J.J., Makam, S.N., Chen, R.J., Masson, P.H. Murphy, A.S., 2004. Variation in expression and protein localization of the PIN family of auxin efflux facilitator proteins in flavonoid mutants with altered auxin transport in Arabidopsis thaliana. Plant Cell 16, 1898-1911.

Peer, W.A., Blakeslee, J.J., Yang, H., Murphy, A.S., 2011. Seven things we think we know about auxin transport. Mol. Plant 4, 487-504.

Povero, G., Gonzali, S., Bassolino, L., Mazzucato, A., Perata, P., 2011. Transcriptiona analysis in high-anthocyanin tomatoes reveals synergistic effect of Aft and atv genes. J. Plant Physiol. 168, 270-279.

Sánchez-Bravo, J., Ortuño, A.M., Botía, J.M., Acosta, M., Sabater, F., 1992. The decrease in auxin polar transport down the lupin hypocotyl could produce the indole-3-acetic acid distribution responsible for the elongation growth pattern. Plant Physiol. 100, 108-114.

Sauer, M., Robert, S., Kleine-Vehn, J., 2013. Auxin: simply complicated. J. Exp. Bot. $64,2565-2577$

Sorce, C., Lombardi, L., Giorgetti, L., Parisi, B., Ranalli, P., Lorenzi, R., 2009. Indoleacetic acid concentration and metabolism changes during bud development in tubers of two potato (Solanum tuberosum) cultivars. J. Plant Physiol. 166, 1023-1033.

Spanò, L., Mariotti, D., Cardarelli, M., Branca, C., Costantino, P., 1988. Morphogenesis and auxin sensitivity of transgenic tobacco with different complements of Ri T-DNA. Plant Physiol. 87, 479-483.
Steffens, B., Rasmussen, A., 2016. The physiology of adventitious roots. Plant Physiol. 170, 603-617.

Suttle, J.C., 1991. Biochemical bases for the loss of basipetal IAA transport with advancing physiological age in etiolated Helianthus hypocotyls. Plant Physiol. $96,875-880$.

Vidoz, M.L., Loreti, E., Mensuali, A., Alpi, A., Perata, P., 2010. Hormonal interplay during adventitious root formation in flooded tomato plants. Plant J. 63, 551-562.

Vidoz, M.L., Mignolli, F., Aispuru, H.T., Mroginski, L.A., 2016. Rapid formation of adventitious roots and partial ethylene sensitivity result in faster adaptation to flooding in the aerial roots (aer) mutant of tomato. Sci. Hortic. Amst. 201, 130-139.

Visser, E.J.W., Cohen, J.D., Barendse, G.W.M., Blom, C.W.P.M., Voesenek, L.A.C.J., 1996. An ethylene-mediated increase in sensitivity to auxin induces adventitious root formation in flooded Rumex palustris Sm. Plant Physiol. 112 1687-1692.

Xu, M., Zhu, L., Shou, H., Wu, P., 2005. A PIN1 family gene, OsPIN1, involved in auxin-dependent adventitious root emergence and tillering in rice. Plant Cell Physiol. 46, 1674-1681

Yamamoto, Y., Kamiya, N., Morinaka, Y., Matsuoka, M., Sazuka, T., 2007. Auxin biosynthesis by the YUCCA genes in rice. Plant Physiol. 143, 1362-1371.

Zazimalova, E., Napier, R.M., 2003. Points of regulation for auxin action. Plant Cell Rep. 21, 625-634. 